University of Nebraska - Lincoln

DigitalCommons@University of Nebraska - Lincoln

\title{
8-2015
}

\section{Gas-phase electron diffraction from laser-aligned molecules}

Jie Yang

University of Nebraska-Lincoln, s-jyang6@unl.edu

Martin Centurion

University of Nebraska-Lincoln, martin.centurion@unl.edu

Follow this and additional works at: https://digitalcommons.unl.edu/physicscenturion

Part of the Atomic, Molecular and Optical Physics Commons, and the Physical Chemistry Commons

Yang, Jie and Centurion, Martin, "Gas-phase electron diffraction from laser-aligned molecules" (2015). Martin Centurion Publications. 24.

https://digitalcommons.unl.edu/physicscenturion/24

This Article is brought to you for free and open access by the Research Papers in Physics and Astronomy at DigitalCommons@University of Nebraska - Lincoln. It has been accepted for inclusion in Martin Centurion Publications by an authorized administrator of DigitalCommons@University of Nebraska - Lincoln. 
Published in Structural Chemistry (2015); doi: 10.1007/s11224-015-0650-4

Copyright (c) 2015 Springer Science+Business Media New York. Used by permission.

Submitted July 2, 2015; accepted July 27, 2015; published online August 19, 2015.

\section{Gas-phase electron diffraction from laser-aligned molecules}

\section{Jie Yang and Martin Centurion}

University of Nebraska-Lincoln, Lincoln, NE 68588, USA

Corresponding author — Martin Centurion, martin.centurion@unl.edu pattern from perfectly aligned molecules contains sufficient information to retrieve the structure directly from the diffraction pattern, without the need for comparison with a theoretical model $[13,14]$. It is, however, so far not possible to experimentally produce perfectly aligned molecules in the gas phase for diffraction experiments. We have recently shown that partial alignment, achieved with a femtosecond laser pulse, is sufficient to retrieve the structure of small molecules in a field-free environment $[15,16]$. While it has not yet been shown that this method can be applied to large molecules, it is clear that diffraction patterns even with partial alignment provide more structural information than diffraction patterns from randomly oriented molecules. Multiple projections of the structure can be recorded by rotating the alignment axis with respect to the direction of the electron beam. An additional advantage of diffraction from aligned molecules is that the modulation depth of the interference fringes increases with alignment, which increases the signal-tonoise ratio in the patterns. Diffraction data from aligned molecules have so far not been incorporated in the standard structure refinement methods of GED.

Molecules can be aligned using intense laser pulses [17]. There have been several experimental demonstrations of electron diffraction from laser-aligned molecules. The alignment breaks the circular symmetry of the diffraction pattern and can thus be detected by quantifying the anisotropy in the patterns. The first experiments showed anisotropy in the diffraction patterns when the molecules are aligned with nanosecond laser pulses [18] and when 
a dissociation reaction is triggered with a femtosecond UV laser pulse $[19,20]$. More recently, diffraction from impulsively aligned molecules has been demonstrated both with electrons [15] and with X-rays [21].

In this manuscript, we show the changes in the diffraction pattern as a result of alignment. We also investigate the temporal evolution of the alignment and the dependence on the laser pulse intensity. Carbon disulfide $\left(\mathrm{CS}_{2}\right)$ is used as a model molecule for this work for the following reasons: (1) The polarizability anisotropy is large, which gives higher degree of alignment; (2) the alignment process is much simpler for linear molecules than for asymmetric-top molecules; and (3) changes in the angular distribution of the molecule are reflected in the diffraction pattern in a manner that is straightforward to interpret. We observe in the diffraction patterns that the alignment increases with laser intensity, in good agreement with simulations, and maps the temporal evolution of alignment on the picosecond timescale.

The remainder of this manuscript is divided in the following sections: Laser alignment of molecules describes the method of impulsive laser alignment; Calculation of diffraction patterns from aligned molecules shows numerical simulations of diffraction patterns with partial and perfect alignment; and Experiments compares experimental and simulated diffraction patterns in addition to data on the temporal evolution of the alignment.

\section{Laser alignment of molecules}

Intense laser light can be used to align nonpolar molecules through an induced-dipole interaction. Laser alignment is a very active field to which many researchers have contributed [17]. Alignment typically requires a high laser intensity, so the use of pulsed lasers is required. In the case of impulsive alignment with femtosecond laser pulses, the laser pulse creates a rotational wave packet via Raman transitions. A higher laser intensity results in higher rotational states being excited and thus can produce a narrower angular distribution. The laser pulses are non-resonant, typically at a wavelength around $800 \mathrm{~nm}$.

The duration of the laser pulses determines whether the alignment process is adiabatic or impulsive. When the pulse duration is longer than the rotational period of the molecule, the alignment is adiabatic. In this case, the degree of alignment follows the intensity envelope of the laser pulse in time; i.e., alignment is highest when the laser intensity is maximal, and the ensemble returns to a random distribution when the laser intensity goes to zero. If the laser pulse duration is much shorter than the rotational period of the molecule, the alignment is impulsive. In the classical picture, an impulsive torque is imparted to the molecules. The torque depends on the orientation of the molecules with respect to the polarization of the laser field, with the maximum torque being for molecules with their dipole moment at a $45^{\circ}$ angle with respect to the laser polarization. The maximum alignment is reached a short time after the interaction with the laser pulse. This classical picture, however, cannot explain the fact that after the initial alignment, there will be periodic revivals. In the quantum mechanical picture, the laser excites a rotational wave packet. After the initial alignment, the different rotational states in the wave packet will dephase and the alignment disappears almost completely. The alignment will then show periodic revivals at multiples of half the rotational period of the molecule, as the rotational states rephase periodically. The revivals will continue as long as there is no decoherence mechanism, such as collisions.

The main advantage of impulsive alignment is that the molecules can be probed in a field-free environment, while the disadvantage is that the alignment is not as sharp as in the case of adiabatic alignment. It is possible to combine the best of both methods by using a shaped laser pulse with a long rise time (adiabatic turn on) and a very fast decay after the maximum intensity (non-adiabatic turn off) [22]. In this case, the molecules are aligned adiabatically to reach a narrow angular distribution, and then, the field is turned off suddenly. This leads to the molecules being transiently aligned when the laser field is switched off, and also to the revivals characteristic of impulsive alignment. This method is more challenging to implement because it requires a laser pulse with a large spectral bandwidth to support the femtosecond turn off and also a very high pulse energy to reach a high intensity during the turn on. Such laser parameters are not available in most laboratories, and can typically only be achieved at low repetition rates that are not suitable for GED experiments where high count rates are needed.

For the purposes of determining molecular structures, it is preferable to capture a diffraction pattern of molecules in a field-free environment, free from the high intensity of the laser pulse that might distort the molecular structure or the diffraction process. For our GED studies, we have used impulsive alignment with a femtosecond laser pulse. A linearly polarized laser pulse produces an alignment of the $\mathrm{CS}_{2}$ molecules along the direction of the laser polarization.

We have simulated the interaction of $\mathrm{CS}_{2}$ molecules with the laser by modeling the molecule as a linear rigid rotor interacting with a non-resonant laser pulse described by the time-dependent Schro" dinger equation [23]. The excitation of the rotational eigenstates in the presence of the laser field was calculated, and the evolution of the angular distribution of the molecules was then obtained from the incoherent sum over all the initial states given by the Boltzmann distribution. The excitation is calculated, while the laser pulse is present (extending 
for four standard deviations of the laser pulse duration after the peak intensity), and then the distribution evolves in a field-free environment. An initial temperature of $30 \mathrm{~K}$ was assumed for the molecules. This temperature can be achieved experimentally using a seeded molecular beam in supersonic expansion. Lower rotational temperatures lead to sharper alignment.

Figure 1 shows the results of the calculation. The laser parameters were set to a laser pulse duration of 200 fs and fluence of $0.58 \mathrm{~J} / \mathrm{cm}^{2}$. The angular distribution of the molecules is characterized by the parameter $\left\langle\cos ^{2} \theta\right\rangle$, where the bracket denotes an ensemble average over all the molecules, and $\theta$ is the angle between the alignment axis (the direction of laser polarization) and the axis of each molecule (the line connecting the two $S$ atoms). A value of $\left\langle\cos ^{2} \theta\right\rangle=0.33$ corresponds to random orientation, while a value of $\left\langle\cos ^{2} \theta\right\rangle=1$ corresponds to perfect alignment. The zero of time in the simulation corresponds to the time when the calculation switches to field free, which in this case is $360 \mathrm{fs}$ after the peak of the laser pulse. The angular distribution already shows some alignment by this time, meaning that there is a significant rotation of the molecules even during the short duration of the laser pulse. The alignment continues to become sharper after the laser pulse, reaching a maximum of $\left\langle\cos ^{2} \theta\right\rangle=0.49$ at a time of 0.9 ps. The first alignment peak has a FWHM of $1.8 \mathrm{ps}$, and thus in order to record a diffraction pattern from aligned molecules, the temporal resolution of the experiment must be on the same timescale or shorter. The time delay to reach maximum alignment and the duration of the alignment both decrease as the laser intensity increases. After the initial alignment, the wave packet dephases rapidly, although the distribution does not go back to random orientation. A trace of alignment remains because the laser breaks the symmetry in the molecular rotations. Periodic revivals of alignment (and anti-alignment) are observed with a time delay of approximately $76 \mathrm{ps}$. Revival corresponds to the molecules aligning again along the direction of the laser polarization, while in anti-alignment, the molecules lie preferentially on a plane with the molecular axes perpendicular to the laser polarization direction. The revivals appear at half the rotational period of the molecule. The rotational constant of $\mathrm{CS}_{2}$ is $B=0.01353 \mathrm{meV}$, and the rotational period is $P=\pi \hbar / B=152.8 \mathrm{ps}$.

\section{Calculation of diffraction patterns from aligned molecules}

In GED experiments, the transverse coherence of the electron beam is larger than the size of the molecules, but it is smaller than the typical distance between molecules in the gas. The diffraction pattern is therefore composed of the incoherent sum of the scattering from individual molecules. The formulas for the diffraction pattern can be simplified for the cases when the molecules are perfectly aligned and for the case where the orientation is random. For the case of partial alignment, one must calculate the pattern due to a single molecule at different orientations with respect to the electron beam and then average the patterns weighting their contributions according to the angular distribution. In order to calculate the diffraction from a single molecule, we assume a molecule at the origin of the coordinate system and an electron beam propagating along the $z$-axis. For a molecule with $N$ atoms, the total scattering intensity as a function of the polar scattering angle and the azimuthal angle $\varphi$ is given by
Figure 1. Simulation of impulsive laser alignment of $\mathrm{CS}_{2}$. In this simulation, the molecules had an initial rotational temperature of $30 \mathrm{~K}$, and the laser pulse had a duration of $200 \mathrm{fs}$ and fluence of $0.58 \mathrm{~J} / \mathrm{cm}^{2}$

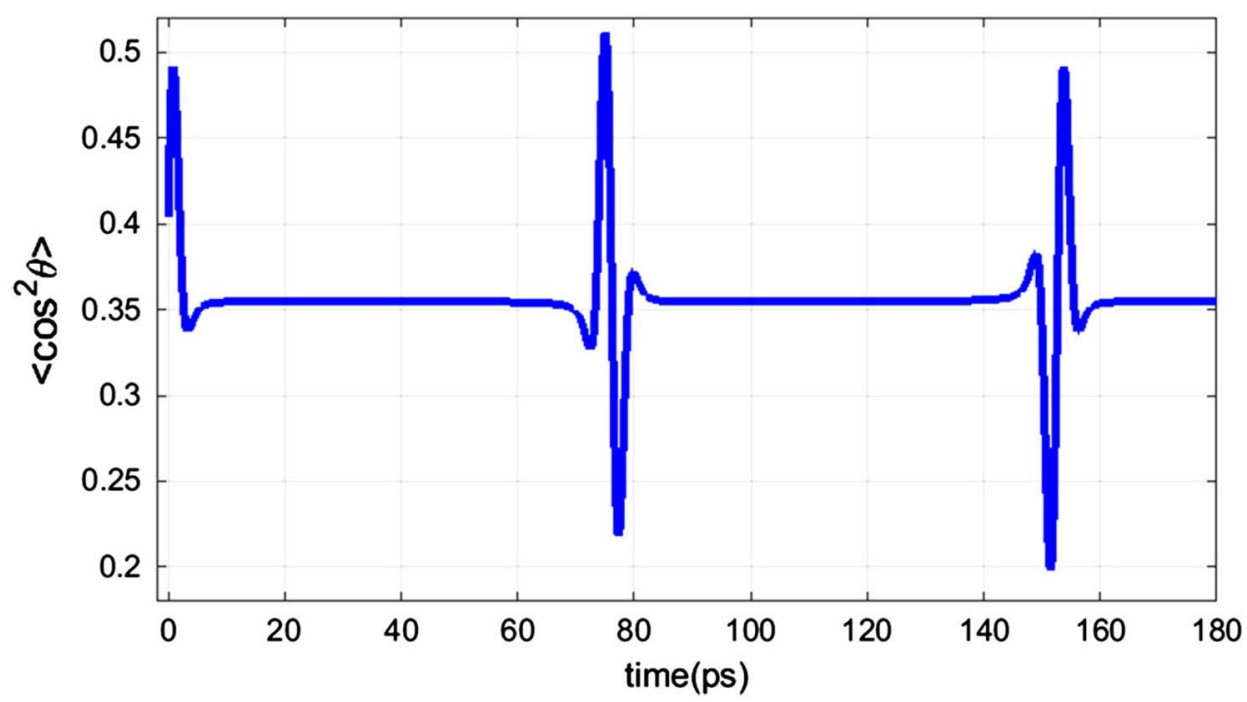




$$
I_{\text {Total }}(\theta, \phi)=\frac{I_{0}}{r^{2}(\theta, \phi)}\left|\sum_{n=1}^{N} f_{n}(\theta) \exp \left(\overrightarrow{i s} \cdot \overrightarrow{r_{n}}\right)\right|^{2}
$$

where $I_{0}$ is a constant, $\vec{r}_{n}$ is the position of the $n$th atom, $\vec{s}=\vec{k}-\vec{k}_{0}$ is the momentum change for diffracted electrons, $r(\mathrm{~h}, /)$ is the distance from the origin to the detector position with angular coordinates $(\theta, \varphi), f_{n}(\theta)$ is the scattering form factor of the $n$th atom, $k=2 \pi / \lambda$ is the wavenumber of the electrons and $\lambda$ is the wavelength. The waves scattered from each atom interfere at the detector, which records the intensity of the wave. Internal vibrations are not included in the model because the bond length changes are beyond the resolution achieved in this work.

Most GED experiments to date have been performed with randomly oriented molecules. After averaging over all possible angular orientations of the molecule, one recovers the familiar formulas for the scattering intensity from randomly oriented gas-phase molecules [1]:

$$
I_{\text {Random }}(s)=\frac{I_{0}}{r^{2}(s)} \sum_{i=1}^{N} \sum_{j=1}^{N} f_{i}(s) f_{j}^{*}(s) \frac{\sin \left(r_{i j} s\right)}{r_{i j} s}
$$

where $s=2 k \sin (\theta / 2)$ is the magnitude of the momentum transfer and the double summations are over all atoms in the molecule. Notice that in this case there is no dependence on the azimuthal angle $\varphi$; i.e., the diffraction patterns will be circularly symmetric.

The total scattering intensity can be split into an atomic and a molecular contribution. Assuming that the distance from the molecules to the detector is much larger than the size of the detector, so that $r$ is approximately constant over the flat surface of the detector, the total intensity becomes:

$$
\begin{aligned}
I_{\text {Random }}(s)= & I_{\text {Atomic }}(s)+I_{\text {Molecular }}(s) \\
I_{\text {Atomic }}(s)= & \frac{I_{0}}{R^{2}} \sum_{i=1}^{N}\left|f_{i}(s)\right|^{2} \\
I_{\text {Molecular }}(s)= & \frac{I_{0}}{R^{2}} \sum_{\substack{i=1 \\
i \neq j}}^{N} \sum_{j=1}^{N}\left|f_{i}(s)\right|\left|f_{j}(s)\right| \\
& \cos \left[\eta_{i}(s)-\eta_{j}(s)\right] \frac{\sin \left(r_{i j} s\right)}{r_{i j} s}
\end{aligned}
$$

where $\left|f_{i}(s)\right|$ and $\eta_{i}$ are the amplitude and phase of the form factor of the ith atom, respectively [24]. I Molecular contains the interference terms and thus the structural information, while $I_{\text {Atomic }}$ depends only on the atomic scattering amplitudes and acts as a background. After the angular averaging due to the random orientation, structural information is still present but only as a weak modulation in the overall scattering intensity $I_{\text {Random }}$ that is measured.

The patterns for partial alignment are simulated following the procedure described in Reference 16. Briefly, the diffraction pattern is first calculated for a single molecule. Then the molecule is rotated and a new diffraction pattern is calculated. Many such patterns are calculated to finely sample all angular orientations. Finally, the patterns are added incoherently, with weights given by the angular distribution produced by the calculation described in the Laser alignment of molecules section.

We have simulated the diffraction patterns of $\mathrm{CS}_{2}$ molecules for various degrees of alignment. Figure 2 shows the total scattering amplitude $\sqrt{ } I_{\text {Total }}$ for four different angular distributions: random orientation, partial alignment with $\left\langle\cos ^{2} \theta\right\rangle=0.50$, partial alignment with $\left\langle\cos ^{2} \theta\right\rangle=0.80$ and perfect alignment. The alignment laser polarization is along the vertical direction. The angular distribution was taken from the simulations at the time of peak alignment. For random orientation (Figure 2a), the pattern is circularly symmetric. When the molecules are aligned, the diffraction pattern becomes anisotropic (Figure 2b, d). For perfect alignment (Figure 2d), the interference fringes can be clearly seen on the pattern. The figure shows the two main features of alignment: the diffraction patterns become anisotropic, and the interference fringes become visible.

The contrast of the interference fringes increases with alignment. Figure 3 shows a vertical cross section (across the center) of the total scattering intensity $I_{\text {Total }}$ as a function of $s$ for the four diffraction patterns shown in Figure 2 .

(a)

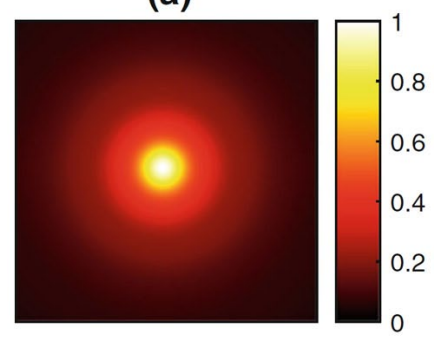

(c)

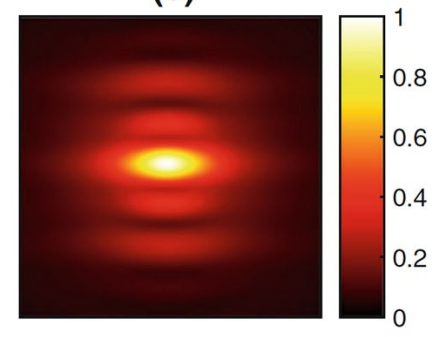

Figure 2. Scattering amplitude $\sqrt{I_{\text {Total }}}$ for a) random orientation, b) $\left\langle\cos ^{2} \theta\right\rangle=0.50$, c) $\left\langle\cos ^{2} \theta\right\rangle=0.80$, and d) perfect alignment. The laser polarization (alignment axis) is vertical in the figure. The figure shows a range of momentum transfer from $s=0 \AA^{-1}$ to $s=7 \AA^{-1}$ in both the horizontal and vertical directions. 
Figure 3. Cross section of the total scattering intensity /Total along the direction of alignment for perfect alignment (blue dashed dotted line), partial alignment with $\left\langle\cos ^{2}\right.$ $\theta\rangle=0.80$ (green dashed line), partial alignment with $\left\langle\cos ^{2} \theta\right\rangle=0.50$ (red dotted line) and random orientation (solid black line).

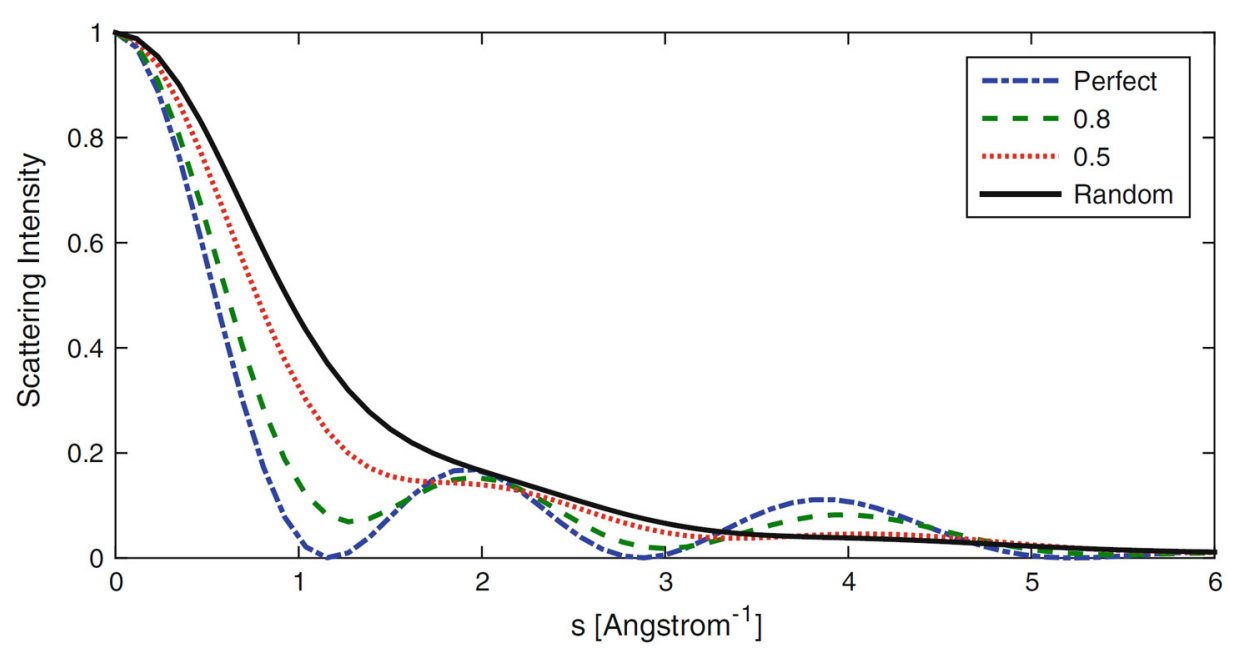

For the case of random orientation, the modulation is not visible without further processing of the signal. The modulation then becomes progressively stronger as the alignment increases, reaching $100 \%$ for perfect alignment (the total intensity reaches zero for certain values of $s$ ). Due to the increased modulation depth, the signal-to-noise ratio for patterns of aligned molecules can be much higher than those of randomly oriented molecules. This could be advantageous for studying more dilute samples or for timeresolved studies where the average electron beam current is low.

Experimentally, it is difficult to achieve a high degree of alignment in a gas jet that is suitable for diffraction experiments. The main difficulty is that very low temperatures $(<1 \mathrm{~K})$ are needed to reach a high alignment, but this low temperature can only be achieved with very low gas densities that are not adequate for diffraction experiments. When the alignment is weak, it can most easily be detected by taking the difference between two diffraction patterns: one where the molecules are aligned and one where the orientation is random. The difference patterns are divided by the atomic scattering intensity to bring up the interference features.

$$
I_{\text {Diff }}=\frac{I_{\text {Aligned }}-I_{\text {Random }}}{I_{\text {Atomic }}}
$$

Figure $4 \mathrm{a}-\mathrm{c}$, shows the scaled diffraction patterns: $I_{\text {Random } / I_{\text {Atomic }},} I_{\text {Aligned } / I_{\text {Atomic }}}$ and $I_{\text {Diff }}$, respectively. The alignment corresponds to $\left\langle\cos ^{2} \theta\right\rangle=0.50$, which can be readily obtained experimentally. The scaling brings up the diffraction rings in Figure 4a. In Figure 4b, the anisotropy due to the alignment can be clearly observed. Finally, in Figure $4 c$, the difference between the two patterns shows a strong anisotropy and high contrast. The advantage of the difference method is that any experimental background is removed when taking the difference.
Figure 5 shows two perpendicular cross sections of $I_{\text {Diff. }}$ The vertical cross section is parallel to the alignment axis, and the horizontal cross section is perpendicular to the alignment axis. This shows that the difference method is very sensitive to bring out the dependence of the diffracted intensity on the azimuthal angle $\varphi$. In fact, even a very small degree of alignment of $\left\langle\cos ^{2} \theta\right\rangle=0.35$ can be reliably detected. GED is a very sensitive method that could be used to determine the angular distribution of molecules.

\section{Experiments}

We have experimentally recorded the diffraction patterns of laser-aligned molecules as a function of the time after the laser excitation, for different values of laser intensity. Figure 6 shows a sketch of the experimental setup. The laser, electron and gas beams are mutually orthogonal. The electron pulses are generated using a photocathode and accelerated in a static field to a kinetic energy of $25 \mathrm{keV}$, which corresponds to $\lambda=7.7 \mathrm{pm}$. The number of electrons per pulse is set to 1000 , which results in a pulse duration of approximately $400 \mathrm{fs}$ at the target. The beam current was measured, and the corresponding pulse duration was calculated using a particle tracer code. The laser pulses have a duration of $200 \mathrm{fs}$, maximum energy of $2 \mathrm{~mJ}$ and a wavelength of $800 \mathrm{~nm}$. The laser operates at a repetition rate of $5 \mathrm{kHz}$. $\mathrm{CS}_{2}$ molecules are seeded into a buffer gas of Helium with a 1:20 ratio for rotational cooling. The de Laval nozzle has a $30 \mu \mathrm{m}$ diameter at the throat and a 90 $\mu \mathrm{m}$ diameter at the exit. A supersonic jet is formed after the nozzle. The nozzle backing pressure is set to 1000 Torr. The $\mathrm{CS}_{2}$ gas density is approximately $5 \times 10^{15}$ molecules/ $\mathrm{cm}^{3}$ in the interaction region, and the rotational temperature is estimated to be around $30 \mathrm{~K}$ [25]. An imaging detector that can detect single electrons is used to capture 
Figure 4. Scaled diffraction patterns

a) $I_{\text {Random }} / I_{\text {Atomic }}$

b) $I_{\text {Aligned }} / I_{\text {Atomic }}$, and

c) $I_{\text {Diff }}$.

The figure shows a range of momentum transfer from $s$ $=0 \AA^{-1}$ to $s=7 \AA^{-1}$ in both the horizontal and vertical directions.

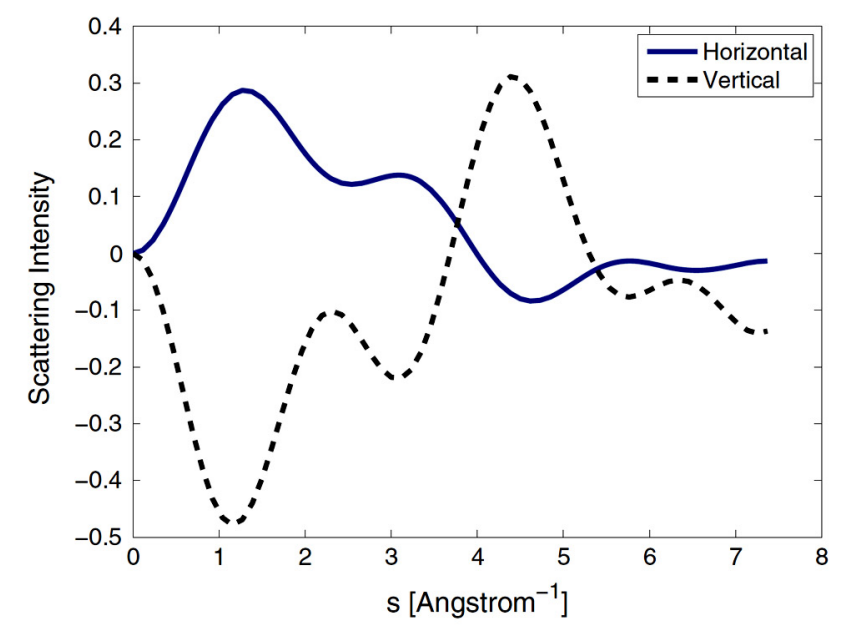

Figure 5. Vertical (dashed black line) and horizontal (solid blue line) cross section of $I_{\text {Diff }}$. The alignment axis is in the vertical direction.

the diffraction patterns. The detector comprises a phosphor screen and an image intensifier that is fiber-coupled to a CCD camera. Data are accumulated for 90 min to obtain diffraction patterns with good signal-to-noise ratio, with the experiment operating at a repetition rate of $5 \mathrm{kHz}$ and 1000 electrons per pulse.

The temporal resolution was limited mainly by the group velocity mismatch between photons and electrons [26]. The width of the gas, laser and electron beams was measured in the overlap region to determine the contribution of velocity mismatch to the resolution. The sizes of electron beam, laser beam and gas jet are $100 \mu \mathrm{m}, 100$ $\mu \mathrm{m}$ and $200 \mu \mathrm{m}$ full width at half maximum (FWHM), respectively. The overall resolution, including group velocity mismatch and the duration of electron and laser pulses, was $1.0 \mathrm{ps}$.

Figure $7 a-c$ shows the measured difference patterns $I_{\text {Diff }}$ for laser fluences of $0.16,0.48$ and $0.79 \mathrm{~J} / \mathrm{cm}^{2}$, respectively. The corresponding laser intensities are $8 \times 10^{11}$, $2 \times 10^{12}$ and $4 \times 10^{12} \mathrm{~W} / \mathrm{cm}^{2}$. The values of the intensity are kept below the ionization threshold. The minimum laser pulse duration that the laser can produce is $40 \mathrm{fs}$, but for these experiments, the pulse duration was (b)

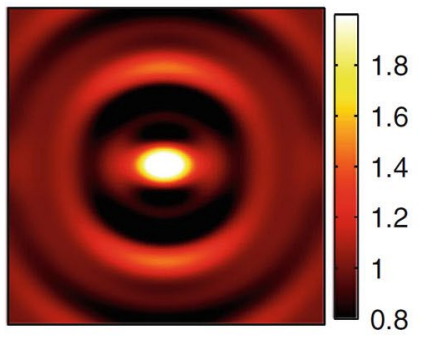

(c)
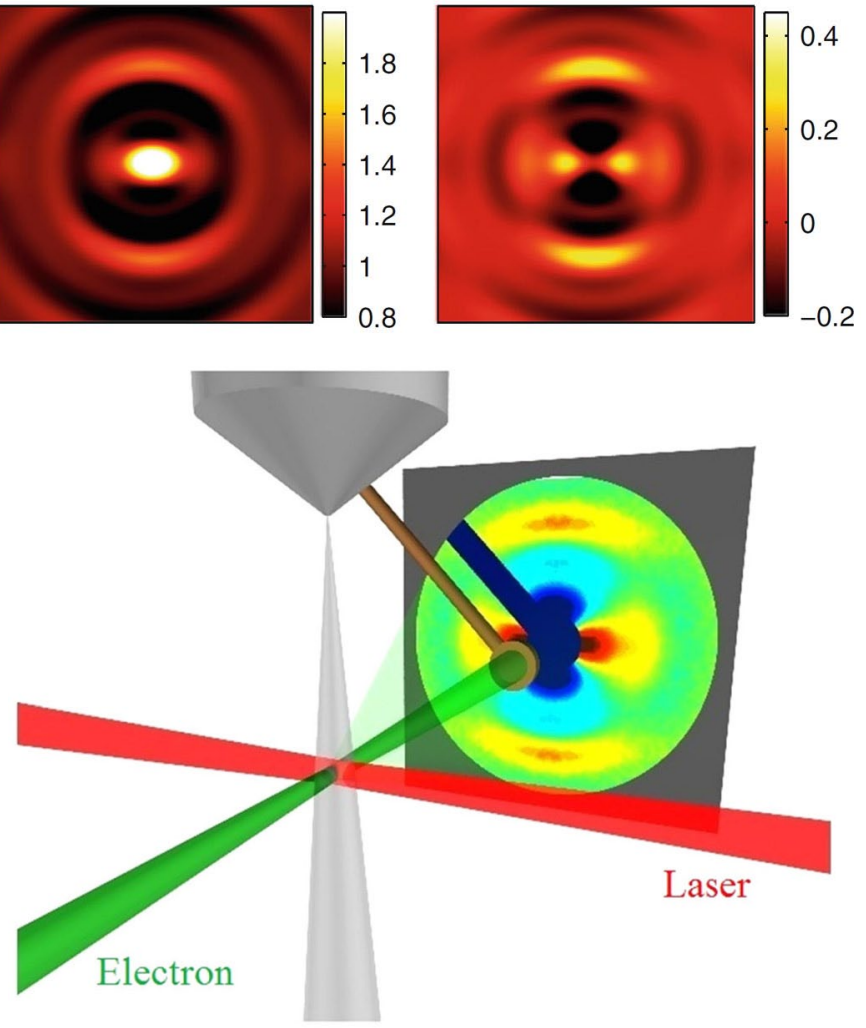

Figure 6. Experimental setup. The electron beam (green), laser beam (red) and gas jet (gray) are mutually orthogonal. The transmitted electron beam (the part that is not diffracted) is blocked to prevent saturation of the detector.

stretched to $200 \mathrm{fs}$ to achieve higher fluence (and thus higher alignment) while keeping the intensity low enough to avoid significant ionization. The pulse duration is still short enough to operate in the regime of impulsive alignment. In a separate study, we have also observed that further increasing the laser intensity does not significantly increase the degree of alignment [27]. The center region ( $s$ $<1.0 \AA^{-1}$ ) of the diffraction patterns is blocked by a beam stop, as shown in Figure 6. In Figure 7a-c, the center of the difference patterns (the part blocked by the beam stop) is filled in by letting the intensity smoothly go to zero toward the center.

Figure $7 d-f$ shows the simulated diffraction patterns. First, the molecular alignment was calculated using the same laser and gas parameters as in the experiment. Then the calculated angular distributions were used to simulate the diffraction patterns. There is very good qualitative and quantitative agreement between experiment and theory. As the laser intensity is increased, the contrast increases significantly in the difference patterns (see the color scale bar next to each image), and it increases in a similar way in experiment and theory. The shape of the diffraction pattern also changes, and the interference fringes become 
(a)

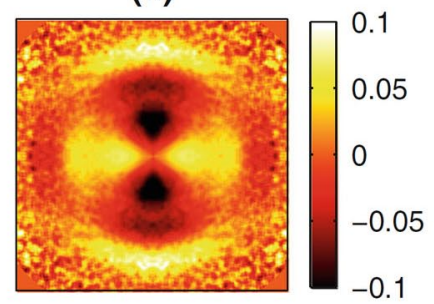

(d)

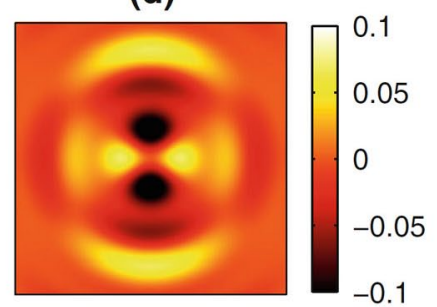

(b)

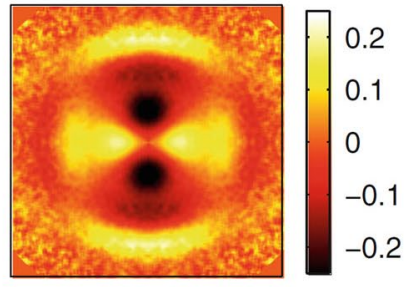

(e)

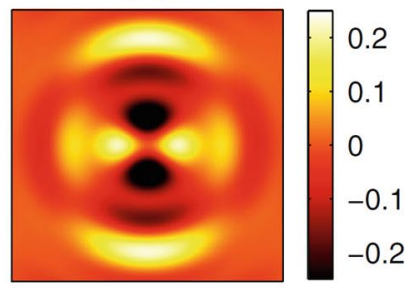

(c)

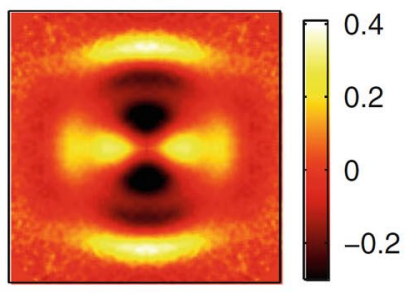

(f)

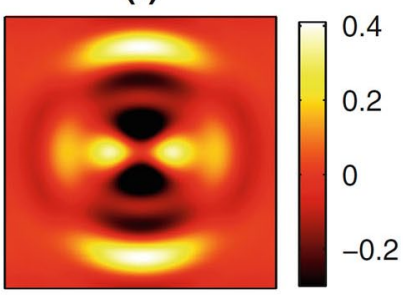

Figure 7. a) - c) Experimental /Diff for laser fluences of $0.16,0.48$ and $0.79 \mathrm{~J} / \mathrm{cm}_{2}$, respectively. d) - f) Patterns simulated using the experimental parameters. The alignment value is $\left\langle\cos ^{2} \theta\right\rangle=0.38$ in $\mathbf{d},\left\langle\cos ^{2} \theta\right\rangle=0.46$ in $\mathbf{e}$, and $\left\langle\cos ^{2} \theta\right\rangle=0.54$ in $\mathbf{f}$. The patterns show a range of momentum transfer from $s=0 \AA^{-1}$ to $s=5.6 \AA^{-1}$ in both the horizontal and vertical directions.

less curved as the alignment increases, showing that the molecules are more tightly aligned. In the simulations, the alignment increases from $\left\langle\cos ^{2} \theta\right\rangle=0.38$ in Figure $7 d$ to $\left\langle\cos ^{2} \theta\right\rangle=0.54$ in Figure $7 f$.

We have also monitored the temporal evolution of the alignment. Experimentally, the alignment can be characterized by the anisotropy in the diffraction pattern. In order to quantify the anisotropy, each pattern is divided in four quadrants: two that are aligned vertically and two horizontally. The boundaries of the quadrants are defined by two straight lines that cross at the center and start and end at opposite corners of the image. The anisotropy is calculated by adding the counts in the range between $s=1 \AA^{-1}$ and $s=3.5 \AA^{-1}$ and is defined as

$$
\text { Anisotropy }=\frac{I_{\text {Horizontal }}-I_{\text {vertical }}}{I_{\text {Atomic }}}
$$

where $I_{\text {Horizontal }}$ and $I_{\text {vertical }}$ are the total counts in the horizontal and vertical quadrants, respectively. In this range, there is a large difference in the intensity of the diffraction rings in the vertical and horizontal directions (see Figure 5).

Figure 8a shows the temporal evolution of the anisotropy as a function of time for three different values of the laser fluence. Figure $8 \mathrm{~b}$ shows the calculated values of $\left\langle\cos ^{2} \theta\right\rangle$ for the same values of laser fluence. Both experiment and simulation show a strong increase in alignment with laser fluence. In both experiment and simulation, the maximum alignment is achieved between 1.2 and $1.5 \mathrm{ps}$ after time zero. By this time, the laser pulse is gone and the molecules are in a field-free environment. The time delay to reach peak alignment decreases as the laser fluence increases and higher rotational states are excited.
The width of the alignment peak is also comparable in theory and experiment when the temporal resolution is taken into account. The full width at half maximum (FWHM) of the experimental measurement for the highest intensity (blue solid line in Figure $8 \mathrm{a}$ ) is $1.6 \mathrm{ps}$. The FWHM of the corresponding theory curve in Figure $8 \mathrm{~b}$ is $1.3 \mathrm{ps}$. The broader peak measured experimentally results from the convolution of the width of the alignment peak with the temporal resolution of the measurement of $1 \mathrm{ps}$. Assuming Gaussian widths, we can estimate that the experimental value should be $\sqrt{1.3^{2}+1.0^{2}}=1.6 \mathrm{ps}$.

After the alignment, the anisotropy does not return to zero. The higher the alignment, the higher the offset from random orientation that remains afterward. This can be seen in both experiment and theory. As mentioned before, it is a feature of the alignment process that the distribution does return to random afterward. It is worth noting that for the intermediate intensity in Figure $8 \mathrm{~b}$ (dashed green curve), the remnant alignment after the peak is very low, $\left\langle\cos ^{2} \theta\right\rangle=0.34$. As can be seen in Figure $8 \mathrm{a}$, even this very small alignment can be detected with good sensitivity using GED.

\section{Summary}

This paper describes the basic mechanisms and features involved in electron diffraction from aligned molecules. We have shown both experimentally and theoretically that it is possible to record diffraction patterns from molecules impulsively aligned by a femtosecond laser pulse. In this case, peak alignment is reached after the laser pulse is gone, and thus, a diffraction pattern can be captured in 
a field-free environment. The alignment produces a clear signature in that the diffraction patterns become anisotropic. Alignment also increases the contrast of the interference in the diffraction pattern. The data in diffraction from partially aligned molecules could potentially be incorporated with the standard procedures for structure refinement of GED, as it can provide additional constraints. One current limitation is the small $s$ range captured in timeresolved diffraction experiments, but that is expected to improve significantly with improved technologies in electron pulse generation and compression $[28,29]$.

Acknowledgments - This work was supported by the US Department of Energy Office of Science, Office of Basic Energy Sciences under Award Number DE-SC0003931.
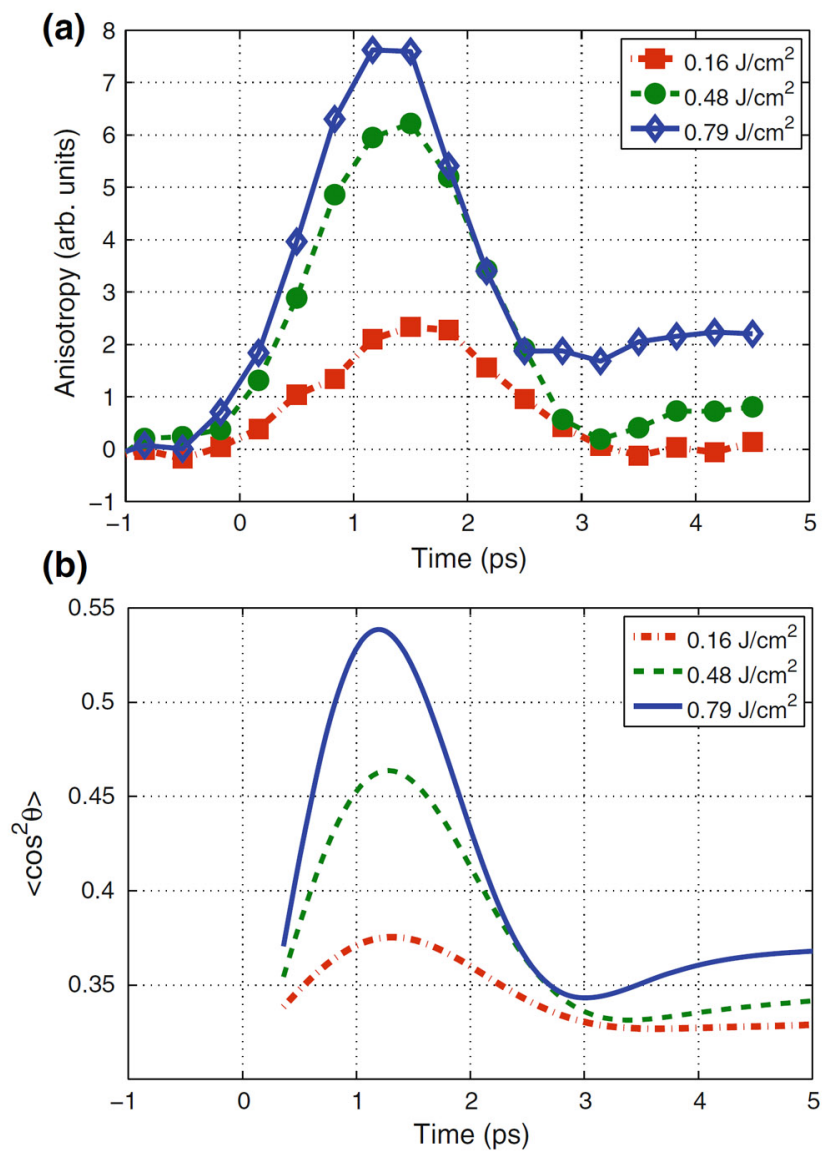

Figure 8. Temporal evolution of alignment. a) Experimental measurement of anisotropy for laser fluences of $0.16 \mathrm{~J} / \mathrm{cm}^{2}$ (red dashed dotted line), $0.48 \mathrm{~J} / \mathrm{cm}^{2}$ (green dashed line) and $0.79 \mathrm{~J} /$ $\mathrm{cm}^{2}$ (blue solid line). b) Simulated degree of alignment for laser fluences of $0.16 \mathrm{~J} / \mathrm{cm}^{2}$ (red dashed dotted line), $0.48 \mathrm{~J} / \mathrm{cm}^{2}$ (green dashed line) and $0.79 \mathrm{~J} / \mathrm{cm}^{2}$ (blue solid line).

\section{References}

1. Hargittai I, Hargittai M (1988) Stereochemical applications of gas-phase electron diffraction. VCH Publishers, New York

2. Varga $Z$, Vest B, Schwerdtfeger P, Hargittai M (2010) Inorg Chem 49:2816-2821

3. Hnyk D, Wann DA, Holub J, Samdal S, Rankin DWH (2011) Dalton Trans 40:5734-5737

4. Schwabedissen J, Lane PD, Masters SL, Hassler K, Wann DA (2014) Dalton Trans 43:10175-10182

5. Kolesnikova IN, Dorofeeva OV, Karasev NM, Oberhammer H, Shishkov IF (2014) J Mol Struct 1074:196-200

6. Wu Z, Li H, Zhu B, Zeng X, Hayes SA, Mitzel NW, Beckers H, Berger RJF (2015) Phys Chem Chem Phys 17:8784-8791

7. Zhabanov YA, Zakharov AV, Giricheva NI, Shlykov SA, Koifman Ol, Girichev GV (2015) J Mol Struct 1092:104-112

8. Romenesko DJ, Wong TC, Bartell L (1975) In: Sim GA, Sutton LE (eds) Molecular structure by diffraction methods. The Chemical Society, London

9. Blom R, Cradock S, Davidson SL, Rankin DWH (1991) J Mol Struct 245:369-377

10. Davis MJ, Rankin DWH, Cradock S (1990) J Mol Struct 238:273-287

11. Klimkowski V, Ewbank JD, Van Alsenoy C, Scarsdale JN, Schäfer L (1982) J Am Chem Soc 104:1476-1480

12. Mitzel NW, Rankin DWH (2003) Dalton Trans 19:3650-3662

13. Spence JCH, Doak RB (2004) Phys Rev Lett 92:198102

14. Pabst S, Ho PJ, Santra R (2010) Phys Rev A 81:043425

15. Hensley CJ, Yang J, Centurion M (2012) Phys Rev Lett 109:133202

16. Yang J, Makhija V, Kumarappan V, Centurion M (2014) Struct Dyn 1:044101

17. Stapelfeldt H, Seideman T (2003) Rev Mod Phys 75:543-557

18. Hoshina K, Yamanouchi K, Takashi T, Ose Y, Todokoro H (2003) J Chem Phys 118:6211-6221

19. Reckenthaeler PR, Centurion M, Fuss W, Krausz F, Fill EE (2009) Phys Rev Lett 102:213001

20. Centurion M, Reckenthaeler P, Krausz F, Fill E (2010) J Mol Struct 978:141-146

21. Küpper J, Stern S, Holmegaard L et al (2014) Phys Rev Lett 112:083002

22. Seideman T (2001) J Chem Phys 115:5965-5973

23. Seideman T, Hamilton E (2005) Adv At Mol Opt Phys 52:289-329

24. Prince E (2004) International tables for crystallography mathematical, physical and chemical tables. Kluwer, Dordrecht

25. Pullman D, Friedrich B, Herschbach D (1990) J Chem Phys 93:3224-3236

26. Williamson JC, Zewail AH (1993) Chem Phys Lett 209:10-16

27. Yang Y, Beck J, Uiterwaal CJ, Centurion M (2015) http://arxiv. org/abs/1410.6429. Accessed 7 July 2015

28. Oudheusden T, Pasmans PLEM, van der Geer SB, de Loos MJ, van der Wiel MJ, Luiten OJ (2010) Phys Rev Lett 105:264801

29. Weathersby SP, Brown G, Centurion M et al (2015) Rev Sci Instrum 86:073702 